\title{
LAS FUENTES DEL CRECIMIENTO ECONÓMICO ESPAÑOL, 1964-1973
}

MAR CEBRIÁN VILLAR *

Universidad Carlos III

\section{RESUMEN}

El principal objetivo de esta investigación es conocer las fuentes del crecimiento del producto real de la economía española en el periodo 1964-1973. Para ello se han distinguido los siguientes componentes: stock de capital, calidad del stock de capital, número de horas trabajadas, calidad de las horas trabajadas y PTF. Se ha utilizado una función de producción Translog y se han diferenciado el crecimiento de la calidad y el crecimiento de la cantidad de los factores productivos. El resultado que se obtiene es que el principal componente del crecimiento del producto es el crecimiento de la PTF.

\section{ABSTRACT}

The aim of this paper is to investigate the sources of growth of real product in the Spanish economy over the period 1964-1973. This is decomposed into five components: capital stock, quality of capital stock, hours worked, quality of hours worked and TFP. The methodology is based on the transcendental logarithmic production function and distinguishes growth in quality and quantity of inputs. We find that the main component of the growth in output is total factor productivity.

\section{INTRODUCCIÓN}

La literatura empírica sobre la contabilidad del crecimiento y la productividad total de los factores (PTF) se basa en establecer los determinantes del crecimiento, tratando de diferenciar el crecimiento de los inputs del crecimiento de la PTF. Pocos trabajos, sin embargo, son capaces de

* Agradezco los comentarios y sugerencias de Leandro Prados de la Escosura, James Simpson, Santiago López y, en especial, de Joan R. Rosés. Asimismo me gustaría agradecer las valiosas apreciaciones de los dos evaluadores anónimos y de los asistentes al Seminario que se celebró en Cuenca los días 29 y 30 de junio de 2000, especialmente los de Carles Sudriá y Albert Carreras.

Revista de Historia Económica

Año XIX, 2001, N. extraordinario. 
separar adecuadamente la influencia, por un lado, de la acumulación de capital y de trabajo y, por el otro, de la calidad de ambos factores de producción ${ }^{1}$. Por lo que respecta al capital, existe un debate entre los economistas sobre el papel que desempeña el progreso técnico incorporado en el capital y aquel que es independiente de la formación de capital. En este artículo se parte de la premisa de que la introducción de nuevos y más eficientes bienes de capital es una fuente importante del cambio productivo, así como también lo es la calidad del input trabajo. Al igual que la bibliografía sobre el cambio de calidad, se supone que el cambio técnico determina buena parte del precio de los bienes de inversión $^{2}$, y que la calidad del trabajo, debida a una mayor acumulación de capital humano, es un determinante de las variaciones en el salario de los trabajadores ${ }^{3}$. Partiendo de este supuesto, se trata de estimar la contribución del cambio tecnológico implícito en el capital y del incremento de la calidad del trabajo, así como la participación de la acumulación de los factores de producción y de la PTF al crecimiento del producto final, básicamente del sector privado. Con ello, se está ajustando el incremento del PIB para reflejar los cambios en la calidad de los inputs, que no se incluyen en el residuo, sino en la contribución al crecimiento de los inputs. Se ofrece además una comparación internacional de las pautas del crecimiento económico entre 1963 y $1973^{4}$, período en el que España experimentó el mayor crecimiento del siglo $\mathrm{xx}$, que permitió un proceso de convergencia. La comparación se realiza entre España y EE UU, Canadá,

${ }^{1}$ Rafael Myro (1983) ha calculado la PTF de la economía española con la misma metodología, pero sin efectuar una comparación internacional. Las diferencias entre el presente trabajo y el suyo residen en la utilización de distintos datos sobre el stock de capital y sobre el output. Esto se traduce en tasas diferentes de crecimiento. Así, por ejemplo, la serie de producción utilizada por Myro crece a un ritmo mayor, pero, según sus datos, el capital crece a un menor ritmo. Para este mismo período, existen otros trabajos sobre las fuentes del crecimiento español aunque con distinta metodología (y no contemplan la calidad de los inputs productivos), véanse, entre otros, Suárez Bernaldo de Quirós (1992); Raymond (1995) para los años 1961-1991; Pérez, Goerlich y Mas (1996) para el periodo 1964-1991; Escribá Pérez y Murgui García (1998) para los años 1980-1993; Maudós, Pastor y Serrano (2000), que descomponen la tasa de crecimiento de la PTF que es debida al cambio técnico y a cambios de eficiencia. Todos estos trabajos concluyen, al igual que éste, que el principal determinante del crecimiento del output ha sido el crecimiento de la PTF.

2 Hulten (1992).

${ }^{3}$ Véase Jorgenson (1996), pp. 23-26.

4 La razón de empezar el análisis en el año 1964 es la ausencia de una estimación sobre el stock de capital en España para el período 1958-1963, así como a las deficiencias en la información estadística sobre salarios y horas trabajadas, sólo disponibles a partir de 1963, gracias a la encuesta Salarios, del INE. 
Francia, Alemania, Italia, Japón, Corea, Holanda y el Reino Unido, países para los cuales se ha aplicado idéntica metodología ${ }^{5}$.

La metodología utilizada para el estudio de la PTF ha sido un enfoque de series temporales, con una función de producción Translog, en el que la PTF se estima como un residuo, usando las técnicas desarrolladas por Dale Jorgenson y sus asociados ${ }^{6}$. Este método tiene la particularidad de permitir descomponer las contribuciones del crecimiento de los inputs capital y trabajo al incremento del output en dos factores principales. El primero está constituido por las aportaciones del stock de capital y de las horas trabajadas, y el segundo por las de la calidad del capital y del trabajo ${ }^{7}$. El input capital sería proporcional al stock de capital si todos los componentes de este factor fueran homogéneos; esto es, si todos tuvieran la misma productividad marginal. Lo mismo ocurriria con el factor trabajo. La calidad de ambos factores se obtiene, por tanto, como la diferencia entre las tasas de crecimiento de los inputs cuando éstos se consideran heterogéneos y las tasas de crecimiento estimadas cuando suponemos que se trata de inputs homogéneos. En resumen, el resultado de este método es una reducción del tamaño de la PTF (al excluir de la misma los cambios en la calidad de los factores de producción) y, simultáneamente, un incremento del peso de ambos factores productivos en el crecimiento del out$p^{2} t^{8}$. A pesar de esta corrección a la baja, esta investigación muestra que el incremento de la PTF, y no el crecimiento de los factores productivos, ha sido la fuente principal en el crecimiento español del período 1963-1973, al menos en lo que respecta al sector privado.

El artículo se compone de dos partes. En la primera se presenta la metodología seguida. Las fuentes, así como los distintos pasos aplicados para la obtención de los datos finales aparecen en el Apéndice (pp. 291-296). En la segunda, se muestran los resultados del estudio para España, y la comparación con una muestra de países. El artículo finaliza

${ }^{5}$ Christensen, Cummings y Jorgenson (1980).

- Esta metodología se basa en el índice Divisia, Translog, también llamado Törnqvist. Este índice asume una función de producción Translog y pertenece al grupo de los llamados indices superlativos (es decir, aquellos que son exactos para una cierta forma funcional. Diewert (1976) demostró que el índice Törnquist es exacto si la función de producción es de la forma Translog homogénea). Estos índices son una aproximación de segundo orden a la función de producción neoclásica, ya que suponen múltiples elasticidades de sustitución entre los factores de producción. Véase Diewert y Nakamura (1993).

7 Jorgenson y Griliches (1967), Jorgenson (1996).

8 En los primeros estudios sobre la contabilidad del crecimiento, en los cuales no se consideraba la calidad de los inputs productivos, la contribución de éstos al crecimiento del output total era tan sólo de un 25 por 100. Véanse Solow (1957) y Denison (1962). 
con unas conclusiones sobre los determinantes principales del crecimiento económico de España en los años sesenta.

\section{METODOLOGÍA}

Consideremos una función de producción Translog (trascendental logarítmica) con rendimientos constantes de escala:

$$
\begin{aligned}
\ln Y=\alpha_{0}+\alpha_{k} \ln K & +\alpha_{L} \ln L+\alpha_{t} t+\frac{1}{2} \beta_{K K}(\ln K)^{2}+\beta_{K L} \ln K \ln L+\beta_{K t} \ln K \\
+ & \frac{1}{2} \beta_{L L}(\ln L)^{2}+\beta_{L} \ln L \times t+\frac{1}{2} \beta_{t t} t^{2}
\end{aligned}
$$

donde $K, L$ y $t$ representan el input capital, el input trabajo y el tiempo, respectivamente, y donde, bajo el supuesto de rendimientos constantes de escala, los parámetros $\alpha_{i}$ y $\beta_{j m}$ cumplen las siguientes condiciones:

$$
\begin{aligned}
& \alpha_{K}+\alpha_{L}=1, \\
& \beta_{K K}+\beta_{K L}=0 \\
& \beta_{K L}+\beta_{L L}=0
\end{aligned}
$$

Sea $P_{Y}$ el precio del output, $P_{K}$ el precio del capital y $P_{L}$ el precio del input trabajo. Las participaciones de los inputs capital y trabajo en la remuneración total de los factores son:

$$
\begin{aligned}
& v_{K}=P_{K} K / P_{Y} Y \\
& v_{L}=P_{L} L / P_{Y} Y \\
& v_{K} \text { y } v_{L} \text { pueden expresarse como: }
\end{aligned}
$$

$$
\begin{aligned}
& v_{K}=\frac{\partial \ln Y}{\partial \ln K}(K, L, t)=\alpha_{K}+\beta_{K K} \ln K+\beta_{K L} \ln L+\beta_{K, t} t \\
& v_{L}=\frac{\partial \ln Y}{\partial \ln L}(K, L, t)=\alpha_{L}+\beta_{K L} \ln K+\beta_{L L} \ln L+\beta_{L t} t
\end{aligned}
$$

Las condiciones necesarias para el equilibrio implican la igualdad entre las participaciones de los inputs en el valor del producto y las correspondientes elasticidades del output con respecto a los inputs. Esto supone que, en equilibrio, los precios de los factores son iguales al valor de sus productividades marginales.

De igual forma, la tasa de cambio técnico es: 


$$
v_{t}=\frac{\partial \ln Y}{\partial \ln t}(K, L, t)=\alpha_{t}+\beta_{K t} \ln K+\beta_{L} \ln L+\beta_{t t} t
$$

Consideremos que tenemos datos para dos períodos de tiempo, por ejemplo, $t$ y $t-1$. La tasa media de crecimiento de la productividad total de los factores puede expresarse como la diferencia entre los sucesivos logaritmos del output menos la media ponderada de las diferencias entre los sucesivos logaritmos de los inputs capital y trabajo, siendo las ponderaciones $\left(\bar{v}_{K}\right.$ y $\left.\bar{v}_{L}\right)$ las participaciones medias de los respectivos inputs en la remuneración total de los factores ${ }^{9}$ :

$$
\begin{aligned}
\ln Y(t) & -\ln Y(t-1)=\bar{v}_{K}[\ln K(t)-\ln K(t-1)]+ \\
& +\bar{v}_{L}[\ln L(t)-\ln L(t-1)]+P T F_{t-1, t}
\end{aligned}
$$

donde:

$$
\begin{aligned}
& \bar{v}_{K}=\frac{1}{2}\left[v_{K}(t)+v_{K}(t-1)\right] \\
& \bar{v}_{L}=\frac{1}{2}\left[v_{L}(t)+v_{L}(t-1)\right]
\end{aligned}
$$

El índice Translog del crecimiento de la PTF $\left(P T F_{t-1, t}\right)$ permite conocer el incremento del output que no es atribuible al crecimiento de los inputs capital y trabajo. Si el capital y el trabajo son funciones Translog de sus componentes, entonces las formas Translog de ambos inputs son:

$$
\begin{aligned}
\ln K=\alpha_{1}^{K} \ln K_{1}+\alpha_{2}^{K} \ln K_{2} & +\ldots+\alpha_{n}^{K} \ln K_{n}+\frac{1}{2} \beta_{11}^{K}\left(\ln K_{1}\right)^{2}+\beta_{12}^{K}\left(\ln K_{1}\right)\left(\ln K_{2}\right) \\
& +\ldots+\frac{1}{2} \beta_{m n}^{K}\left(\ln K_{n}\right)^{2} \\
\ln L=\alpha_{1}^{L} \ln L_{1}+\alpha_{2}^{L} \ln L_{2} & +\ldots+\alpha_{m}^{L} \ln L_{m}+\frac{1}{2} \beta_{11}^{L}\left(\ln L_{1}\right)^{2}+\beta_{12}^{L}\left(\ln L_{1}\right)\left(\ln L_{2}\right) \\
& +\ldots+\frac{1}{2} \beta_{m m}^{L}\left(\ln L_{m}\right)^{2}
\end{aligned}
$$

Las diferencias entre los sucesivos logaritmos nos dan las tasas de crecimiento de los inputs capital y trabajo y tienen la siguiente expresión:

` Para un desarrollo más completo, véase Christensen, Cummings, Jorgenson (1980). 


$$
\begin{aligned}
& \ln K(t)-\ln K(t-1)=\sum_{i} \bar{v}_{K_{i}}\left[\ln K_{i}(t)-\ln K_{i}(t-1)\right] \rightarrow \begin{array}{l}
\text { Índice Translog del } \\
\text { input capital. }
\end{array} \\
& \ln L(t)-\ln L(t-1)=\sum_{j} \bar{v}_{L_{i}}\left[\ln L_{j}(t)-\ln L_{j}(t-1)\right] \rightarrow \rightarrow \begin{array}{l}
\text { Indice Translog del } \\
\text { input trabajo. }
\end{array}
\end{aligned}
$$

donde:

$$
\begin{aligned}
& \bar{v}_{K i}=\frac{\left[v_{K i}(t)+v_{K i}(t-1)\right]}{2}(i=1,2, \ldots, n) \\
& \tilde{v}_{L i}=\frac{\left[v_{L i}(t)+v_{L i}(t-1)\right]}{2}(j=1,2, \ldots, p)
\end{aligned}
$$

$\bar{v}_{i j}$ representa la elasticidad de cada input agregado respecto de cada uno de los subinputs componentes o, lo que es lo mismo, si suponemos competencia perfecta, la participación de cada subinput en la remuneración de los inputs respectivos. Ambos índices permiten tener en cuenta las mejoras que se producen como consecuencia de la calidad de los inputs agregados capital y trabajo, ponderando el crecimiento de cada subinput por su productividad marginal.

La medida correcta del capital como un factor de producción son los servicios que dicho capital ofrece. Se supone que los servicios del capital son proporcionales al stock de capital neto existente a finales del año anterior ${ }^{10}$. Ahora bien, no todo el capital suministra los mismos servicios, sino que éstos varían en función de los tipos de capital, puesto que el progreso tecnológico está en parte vinculado a las mejoras en el diseño de la nueva maquinaria y bienes de equipo ${ }^{11}$. En este artículo, como ya se ha afirmado en la introducción, se parte de la premisa de que la introducción de nuevos, y más eficientes, bienes de capital es una fuente importante del cambio productivo ${ }^{12}$. Una consecuencia de la hipótesis del cambio técnico incor-

${ }^{10}$ El capital neto es la diferencia entre el capital bruto y la pérdida de valor de éste. En el presente trabajo se supone, siguiendo a Hulten $(2000)$, p. 8, que la pérdida de la capacidad productiva del stock de capital puede deberse tanto a la depreciación como a los activos retirados.

1 Hulten (1992).

${ }^{12}$ Greenwood, Hercowitz y Krusell (1997). Denison afirmó que el cambio técnico incorporado en los bienes de capital tenía solamente un pequeño impacto en el crecimiento del input, incluso en el supuesto de que todo el cambio tecnológico estuviera implícito en el capital, Denison (1962). Otros autores, como Phelps (1962), también consideran que 
porado en el capital es que el nuevo capital es más productivo que el antiguo ${ }^{13}$. El índice de la calidad del stock de capital se define, por tanto, como la diferencia entre el índice ponderado, esto es, el índice Translog del input capital (ecuación 5), y el índice Translog del stock de capital (esto es, sin ponderar las distintas clases de capital en función de su productividad marginal). La calidad aumenta cuando los componentes del stock de capital con mayores servicios productivos crecen más deprisa que otros elementos del stock de capital. El no ajustar el input capital mediante el cambio de calidad lleva a englobar los efectos de la calidad del capital dentro del residuo o productividad total de los factores. La estrategia para cuantificar la contribución al crecimiento económico del progreso tecnológico implícito en la inversión de capital es usar datos sobre el precio del stock de capital como una medida de dicho progreso tecnológico.

Del mismo modo que en el caso del input capital, se supone también que, para cada uno de los componentes del input trabajo, los servicios productivos son proporcionales al stock de horas de trabajo. Ahora bien, los servicios de trabajo por período de tiempo varían en función del subinput trabajo. Las diferencias en los servicios obtenidos de las horas de trabajo se deben a las distintas remuneraciones del trabajo por hora o, dadas las condiciones de equilibrio en que se basa el modelo, a las diferencias en el valor de las productividades marginales de las horas totales trabajadas. De acuerdo con la teoría del capital humano, las diferencias de productividad entre individuos son básicamente adquiridas por medio de procesos educativos y de formación profesional. Por tanto, al hacer referencia a las diferencias en la productividad nos estamos refiriendo a su vez a los cambios en la calidad del trabajo ${ }^{14}$. Definimos el índice de la calidad del input trabajo como aquel que transforma el índice Translog de las horas trabajadas (esto es, sin ponderar) en un índice Translog del input trabajo (es decir, ponderado). Este índice refleja los cambios en la composición por categorías del total de horas trabajadas, debido a que no todas las horas de trabajo ofrecen los mismos servicios productivos.

la calidad del capital, consecuencia de la incorporación del cambio técnico, es una variable poco importante.

${ }^{13}$ Hulten (1992), p. 965.

${ }^{14} \mathrm{La}$ importancia del capital.humano en el crecimiento económico ha sido destacada por numerosos autores, entre ellos, los premios Nobel de Economía T. W. Schultz y G. S. Becker. Trabajos actuales sobre el tema son, entre otros, los de Romer (1986), Lucas (1990); Mankiw, Romer y Weil (1992), y Barro y Sala i Martín (1992). 


\section{RESULTADOS}

En los siguientes cuadros se presentan unas comparaciones del crecimiento del output, de la PTF, y de los inputs capital y trabajo (tanto del incremento de la cantidad como de la calidad). En el Cuadro 1 puede observarse el crecimiento medio anual de estas cuatro variables para la muestra completa de países.

En cuanto al crecimiento del output (véase el Cuadro 1 y el Cuadro 1 del Apéndice) hay que mencionar a Japón y Corea, con cifras muy superiores a las del resto de países, 10,9 y 9,7 por 100 respectivamente, para el período 1960-1973. España registra una tasa media de crecimiento del 6,2 por 100, muy similar a la de Francia y Holanda. En cuanto a la PTF, el país con una mayor tasa de crecimiento de la PTF es Japón, seguido de España, Corea e Italia (Cuadro 1 y Cuadro 4 del Apéndice).

$\mathrm{Si}$ analizamos el crecimiento de los inputs (Cuadro 1 y los Cuadros 2 y 3 del Apéndice) se obtienen los siguientes resultados. La tasa media de crecimiento del factor trabajo (la suma de las tasas de crecimiento de la calidad del trabajo y de las horas trabajadas) en España en el período 1964-1973 es de 0,3 por 100 , superior tan sólo a las registradas en el período 1960-1973 por Gran Bretaña, Alemania e Italia (estos dos últimos países registran tasas negativas de crecimiento). Destaca Corea con una tasa media del 5 por 100, Holanda con un 3 por 100 y Japón con un 2,7 por 100. Si miramos ahora las tasas anuales puede observarse que España es de los países que presentan las cifras más bajas, con tasas negativas en cuatro de los diez años estudiados. El crecimiento del input trabajo, aunque muy pequeño, se debe al incremento de la calidad de este factor de producción $(0,8$ por 100$)$, ya que las horas trabajadas caen a lo largo del período ( $-0,5$ por 100 , véase Cuadro 2 ). Además del lento crecimiento del factor trabajo, la contribución de éste al crecimiento del output en España es también muy baja, un 3,0 por 100 , aunque superior a las de Italia, Gran Bretaña y Alemania (Cuadro 3). No hay que olvidar que en este período tuvo lugar un importante cambio en la organización del trabajo; este hecho se recoge en la variable PTF; por tanto, no es de extrañar que la contribución del crecimiento del input trabajo sea tan pequeña, puesto que esta variable, tal y como ha sido definida en este trabajo, no está contemplando la mejora en la organización del trabajo.

En cuanto a la tasa media de crecimiento del input capital (la suma de las tasas de crecimiento de la calidad del capital y del stock de capital), son Japón y Alemania los países que registran las cifras más altas, con 


\section{CUADRO 1}

Tasas de crecimiento medio anuales del output, inputs y PTF, 1960-1973 (porcentajes)

\begin{tabular}{lrrrrrrrrrr}
\hline \multicolumn{1}{c}{ Canadá Francia Alemania } & Italia & Japón Corea & Holanda & $\begin{array}{c}\text { Gran } \\
\text { Bretaña }\end{array}$ & EE UU & $\begin{array}{c}\text { España } \\
\text { (1964-73) }\end{array}$ \\
\hline Output .............. & 5,1 & 5,9 & 5,4 & 4,8 & 10,9 & 9,7 & 5,6 & 3,8 & 4,3 & 6,2 \\
Factor capital... & 4,9 & 6,3 & 7,0 & 5,4 & 11,5 & 6,6 & 6,6 & 4,6 & 4,0 & 6,1 \\
Factor trabajo ... & 2,0 & 0,4 & $-0,7$ & $-0,7$ & 2,7 & 5,0 & 3,0 & 0,0 & 2,2 & 0,3 \\
PTF................. & 1,8 & 3,0 & 3,0 & 3,1 & 4,5 & 4,1 & 2,6 & 2,1 & 1,3 & 4,2 \\
\hline
\end{tabular}

FuENrE: Christensen, Cummings y Jorgenson (1980); para España, véase Apéndice.

el 11,5 y el 7 por 100 , respectivamente. España tiene una tasa del 6,1 por 100, muy parecidas a las de Corea, Holanda y Francia $(6,6 ; 6,6$ y 6,3 por 100 , respectivamente, Cuadro 1). Se observa, además, que el capital crece a un mayor ritmo que el output en todos los países, excepto en Canadá, Corea y EE UU, y es prácticamente igual en España (6,1 por 100 frente a un 6,2 por 100). Dicho crecimiento (esto es, el índice Translog del crecimiento de este input) incrementa, aunque muy poco, la tasa de crecimiento del stock de capital que es de un 5,7 por 100 (Cuadro 2). Ello es debido, como veremos más adelante, a la escasa importancia que ha tenido el crecimiento de la calidad del capital en la economía española en los años del llamado «milagro económico».

El Cuadro 3 muestra claramente que la principal fuente del crecimiento económico español en los sesenta fue el crecimiento de la productividad total de los factores (véase el Cuadro 4 del Apéndice para las tasas anuales de crecimiento) ${ }^{15}$. Esto tiene su lógica, ya que, según los estudios sobre

15 La elevada participación de la PTF en el crecimiento de la economía española, a pesar de haber deducido de la misma la calidad de los factores productivos, puede venir explicada por el hecho de no haber contemplado buena parte del sector público y, por tanto, del sector servicios (otros trabajos, como los ya citados anteriormente, también dan porcentajes del 60-65 por 100 , pero engloban al conjunto de la economía). Ello indicaría que el sector privado español de los años 1964-1973 se caracterizó por una elevada productividad total de los factores, superior a la del sector público, véase el trabajo de Sanchis Llopis (1997) para una comparación de las distintas productividades. Por su parte, Bandrés (1995) ha señalado la mayor productividad del capital fijo de las empresas frente a los equipamientos públicos e inmuebles residenciales. También las infraestructuras (no incluidas aquí) tienen un gran impacto sobre la productividad, pero éstas no empezaron su gran crecimiento hasta 1970 , representando únicamente un 9,5 por 100 de la formación bruta de capital fijo en el período 1964-1970; véase también Martínez Serrano y Muñoz (1995) 


\section{CUADRO 2}

Tasas de crecimiento medio anuales del stock $y$ de la calidad del capital $y$ de las horas trabajadas, 1960-1973 (porcentajes)

\begin{tabular}{|c|c|c|c|c|c|c|c|c|c|c|}
\hline & Canada & Francia & Alemania & Italia & Japón & Corea & Holanda & $\begin{array}{c}\text { Gran } \\
\text { Bretaña }\end{array}$ & $E E U U$ & $\begin{array}{c}\text { España } \\
(196473)\end{array}$ \\
\hline \multicolumn{11}{|l|}{ Calidad del stock } \\
\hline Stock de capital. & 3,8 & 5,1 & 6,6 & 5,0 & 8.5 & 39 & 46 & 42 & 30 & 57 \\
\hline $\begin{array}{l}\text { Calidad de las } \\
\text { horas traba- } \\
\text { jadas................. }\end{array}$ & 0,5 & 0,4 & 0,1 & 1,3 & 0,6 & 1,2 & 0,5 & 0,6 & 0,8 & 0,8 \\
\hline $\begin{array}{r}\text { Horas trabaja- } \\
\text { das...................... }\end{array}$ & 1,5 & 0,0 & $-1,0$ & $-2,0$ & 2,2 & 3,8 & $-0,2$ & $-0,6$ & 1,4 & $-0,5$ \\
\hline
\end{tabular}

FUENTE: Christensen, Cummings y Jorgenson (1980); para España, véase Apéndice.

el crecimiento y convergencia de los países, cuanto más atrasado sea un país, mayor será el ritmo de crecimiento de la PTF. Esta conclusión coincide con los resultados obtenidos por De la Fuente, para quien la convergencia de la economía española durante los años sesenta y primera mitad de los setenta se debió, en buena parte, a la difusión internacional de la tecnología ${ }^{16}$. Una de las razones de la elevada PTF podría estar, por tanto, en la posibilidad que tuvo España de copiar la tecnología de los países más avanzados. Aunque no debemos olvidar otros factores que también se engloban en la PTF, como los gastos de I + D, los cambios organizativos, las economías externas, o los errores de estimación. La segunda fuente más importante en el crecimiento económico español de este periodo fue el crecimiento del capital, con una contribución del 31,7 por 100 , una cifra aparentemente muy importante, pero pequeña en relación con la de otros países (Cuadro 3).

Pasemos a continuación a analizar la contribución de la calidad de los inputs productivos. Los cambios en la calidad del input trabajo se deben a la distinta productividad de las horas trabajadas en las diversas ramas de actividad, y los del input capital están asociados al progreso técnico

y Pérez, Goerlich y Mas (1996). Asimismo, Myro y Gandoy (1995) ya señalaron la mayor productividad del sector industrial (el motor del desarrollo económico en el decenio de 1960 y la primera mitad de los años setenta), frente a la del sector servicios.

${ }^{16}$ Véase también Raymond (1995), p. 98. 


\section{CUADRO 3}

Contribución del crecimiento de los inputs $y$ de la PTF al crecimiento del output, 1960-1973 (porcentajes)

\begin{tabular}{lrrrrrrrrrr}
\hline $1960-73$ & Canada Francia Alemania & Italia & Japón Corea Holanda & $\begin{array}{c}\text { Gran } \\
\text { Bretaña }\end{array}$ & EE UU & $\begin{array}{c}\text { España } \\
(1964,73)\end{array}$ \\
\hline Factor capital... & 43,0 & 44,4 & 52,0 & 43,5 & 43,7 & 25,0 & 50,9 & 46,8 & 39,3 & 31,7 \\
Factor trabajo.... & 20,9 & 4,3 & $-7,4$ & $-9,0$ & 14,7 & 32,9 & 3,1 & $-0,6$ & 30,6 & 3,0 \\
PTF................ & 36,1 & 51,3 & 55,6 & 65,9 & 41,4 & 42,9 & 46,0 & 53,8 & 30,1 & 65,3 \\
\hline
\end{tabular}

FUENTE: Christensen, Cummings y Jorgenson (1980); para España, véase Apéndice.

incorporado en los bienes de capital. La tasa de crecimiento de la calidad de las horas trabajadas para el caso español es, según se observa en el Cuadro 2, de un 0,8 por 100 , inferior a las de Corea e Italia (1,2 y 1,3 por 100 , respectivamente). La calidad del stock de capital crece a una tasa del 0,4 por 100 entre 1964-1973, al igual que en Italia y Gran Bretaña, como puede observarse en el citado cuadro. Esta baja cifra puede deberse a que España invirtió en industrias maduras, de tecnología débil, como ya afirmó Julio Segura, y, por tanto, no de un elevado nivel tecnológico. La productividad del stock de capital no depende únicamente de la calidad de los bienes de equipo, sino también de su buena utilización, organización y comprensión ${ }^{17}$. Además, este resultado coincide con las afirmaciones de Denison y Phelps sobre el pequeño impacto que tiene el cambio técnico incorporado en los bienes de capital en el crecimiento del input ${ }^{18}$. Los mayores crecimientos corresponden a Japón y Corea, con el 3 y el 2,7 por 100 respectivamente. En el caso español, el crecimiento del cambio técnico incorporado (incremento de la calidad) supone un 1,9 por 100 del crecimiento del output en el período 1964-1973 (10,2 por 100 es la cifra para Corea, 11,4 por 100 para Japón, 3,2 por 100 en el caso italiano, Cuadro 4). En cuanto a la contribución del crecimiento de la calidad del trabajo al crecimiento del output destaca la elevada cifra de Italia y EE UU, seguidas de Gran Bretaña y España (véase Cuadro 4). En el caso español,

17 En el crecimiento económico español del periodo 1964-1973, por tanto, más importante que la calidad de la maquinaria (progreso técnico incorporado) fue el cambio técnico desincorporado (aquel que aumenta la productividad de los factores productivos independientemente de la calidad de los mismos, básicamente gracias a una mejor organización del trabajo y a la mejor utilización de la maquinaria y a nuevos sistemas de producción).

is Denison (1964), Phelps (1962). 


\section{CUADRO 4}

Contribución del crecimiento de la calidad y cantidad de los factores productivos en el crecimiento de producto

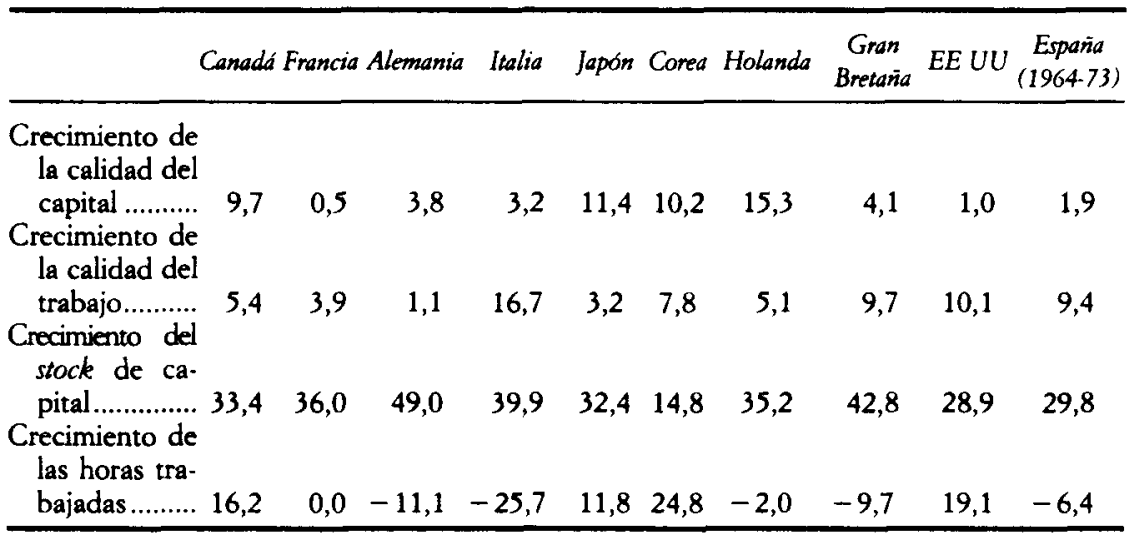

FUENTE: Calculado a partir de Christensen, Cummings y Jorgenson (1980); para España, véase Apéndice.

se observa además un lento crecimiento de la calidad total de los factores, siendo su contribución total al crecimiento económico de un 11,3 por 100 , una cifra baja pero no despreciable.

Si comparamos España con Italia, el país de la muestra más parecido, se observa que España se diferencia en una menor contribución del ritmo de acumulación de capital al crecimiento español, un 31,7 por 100 frente a un 43,5 por 100 en el caso italiano (Cuadro 3) ${ }^{19}$. El trabajo de De la Fuente ya señaló que la tasa de inversión en capital físico en España era significativamente inferior al promedio de la OCDE durante el período 1960-1995 ${ }^{20}$. El crecimiento del input trabajo, en cambio, aunque muy lento, ha sido más importante en el crecimiento económico español de los años sesenta que en el italiano (con una contribución negativa) ${ }^{21}$. A

${ }_{19}$ Véase Prados de la Escosura (1992) para una comparación del crecimiento económico entre España e Italia.

${ }^{20}$ De la Fuente (1998), pp. 13-14.

${ }^{21}$ El haber dejado fuera de esta investigación a la mayor parte de los trabajadores del sector servicios, dada la ausencia de datos sobre las horas trabajadas y los salarios, está sesgando hacia abajo esta cifra y, quizás, en una cantidad no despreciable. La razón de esta afirmación está en que durante el período objeto de estudio la población empleada en el sector servicios es la que experimentó un mayor crecimiento: así, la tasa media anual 
su vez, la contribución del crecimiento de la calidad del trabajo ha sido muy superior en Italia respecto de España (16,7 por 100 frente a un 9,4 por 100 , Cuadro 4), consecuencia del mayor esfuerzo realizado por aquel país en materia de educación.

\section{CONCLUSIONES}

La primera conclusión de esta investigación es que el crecimiento económico español de los años del milagro económico se ha debido sobre todo a la mejora de la productividad total de los factores, con una tasa de crecimiento del 4,2 por 100 entre 1964-1973. Según algunos estudios, el crecimiento que se ha dado en España durante los años sesenta y la primera mitad de los setenta se debió, en buena medida, a un proceso de convergencia, muy relacionado con mecanismos como la difusión tecnológica, las economías externas, la asistencia técnica, el nuevo modo de producción de las fábricas, una nueva organización del trabajo o un cambio tecnológico, variables que recoge la PTF ${ }^{22}$. Por tanto, el proceso de crecimiento y convergencia de la economía española en los sesenta se consiguió esencialmente a través de una mejora en la productividad total de los factores; muy importante fue también la acumulación de capital físico.

Segundo, la contribución del input trabajo al crecimiento económico español de los años sesenta fue muy pequeña. La explicación reside en la disminución en el número de horas trabajadas en algunos sectores, así como en el menor número de trabajadores en algunas categorías socioprofesionales (peones y aprendices y pinches, no asalariados en la industria y trabajadores agrícolas). Otra explicación podría estar en que la base utilizada para el cálculo del número de trabajadores apenas contempla el sector servicios, lo que supone una infravaloración del factor trabajo. Tercero, el crecimiento del capital humano ha sido más importante en el crecimiento del output que el crecimiento de la calidad del capital (un 9,4 por 100 frente a un 1,9 por 100$)^{23}$.

de crecimiento fue del 2,7 por 100 frente al 1,4 por $100,2,6$ por 100 y -3 por 100 en los casos de la industria, construcción y agricultura.

${ }^{22}$ Comín (1995), De La Fuente (1995) y Prados de la Escosura (1999). Los estudios de Baumol (1986), Maddison (1987) y Dowrich y Nguyen (1989), entre otros, también confirman la existencia de convergencia condicional entre los países de la OCDE, debida en gran medida a la difusión tecnológica.

${ }^{23}$ La importancia del capital humano en el crecimiento español ha sido también destacada por numerosos autores, entre ellos, Pérez, Goerlich y Mas (1996). 
Además, la contribución del factor capital, aunque importante (no olvidemos que el 31,7 por 100 del incremento del producto final se debe al crecimiento de la acumulación de capital, tanto nacional como de importación), ha sido baja en relación con países con similar nivel de desarrollo, como es el caso italiano, consecuencia del menor esfuerzo español en la acumulación de capital. Por otro lado, puede afirmarse que la tecnología incorporada en maquinaria importada, dado el bajo ritmo de crecimiento de la calidad del factor capital, no fue un factor clave en el período de mayor crecimiento de la economía española del siglo xx. Mucha mayor relevancia tuvieron los factores, anteriormente citados, que forman parte de la PTF y que han permitido que España convergiera en el período 1964-1973.

Para finalizar haré un comentario respecto a la poca importancia que ha tenido en el crecimiento español de los años sesenta el cambio técnico incorporado en los bienes de capital. La explicación puede residir en la distinta naturaleza del progreso tecnológico en el siglo $\mathrm{xx}$ frente a los siglos anteriores. Como señala Abramovitz, es conveniente distinguir los distintos tipos de cambios tecnológicos que se presentan en cada momento del tiempo ${ }^{24}$. Así, en el siglo $\mathrm{xIX}$, una de las principales fuentes de crecimiento fue la acumulación de capital tangible, ya que el progreso técnico fue intensivo en capital y asociado a la gran escala. Para aplicar cualquier tecnología era preciso aumentar el capital físico por trabajador. Sin embargo, en el siglo $\mathrm{xx}$ se experimentó un cambio hacia un progreso técnico intensivo en capital intangible, que incluye tanto el capital humano como el conocimiento; estos dos factores son considerados como parte del capital por las nuevas teorías del crecimiento endógeno; con lo cual, el concepto de capital es más amplio, ya que no hace referencia solamente al capital físico. Aquella transformación explica la gran importancia que ha adquirido la educación y la acumulación de otros tipos de capital intangibles en el crecimiento de la productividad que han registrado los países durante el siglo $\mathrm{xx}^{25}$. La tecnología de la producción en masa, que se difundió en la economía española en los sesenta, era todavía una tecnología intensiva en capital, al igual que las del siglo XIX, pero era menos intensiva en capital incorporado que las tecnologías del siglo anterior. La explicación está en que al capital (la máquina) hay que «incorporarle» los conocimientos no sólo para hacerla funcionar, como en el siglo xIX, sino para insertar a la

\footnotetext{
${ }^{24}$ Abramovitz (1993).

25 Véase Abramovitz (1993).
} 
maquinaria dentro de un sistema de producción, que hay que diseñar, lo que se considera también tecnología desincorporada ${ }^{26}$. La otra fuente principal de convergencia, como ha afirmado De la Fuente, ha sido el proceso de catch-up que ha experimentado la economía española por partir de niveles relativamente más bajos de ingreso por habitante, que revelan el desfase tecnológico frente al exterior. El hecho de que un país atrasado tienda a crecer a un mayor ritmo que la media es debido a la difusión tecnológica y a la capacidad para adoptar las técnicas de los países más avanzados.

En conclusión, con este artículo se refuerza la posición teórica que prima la importancia de la productividad total de los factores como factor de crecimiento. Y ello, a pesar de haber eliminado del residuo la mejora de productividad debida a la calidad de los factores productivos (que se ha considerado como una parte de la contribución del crecimiento de los insumos capital y trabajo). Por otro lado, este artículo demuestra la afirmación que hiciera Denison en su día, sobre el pequeño impacto que tiene el cambio técnico incorporado en los bienes de capital en el crecimiento del output; al menos en el crecimiento económico español del período más próspero del siglo $\mathrm{xx}$.

\section{APÉNDICE}

El concepto que se ha utilizado para hallar el crecimiento del output ha sido el del producto interior bruto al coste de los factores ${ }^{27}$. La fuente utilizada para el cálculo del crecimiento del output ha sido la estimación ofrecida por Leandro Prados de la Escosura ${ }^{28}$.

El primer paso para el cálculo del input capital es la obtención del stock de capital neto, dato que ha sido extraído del trabajo realizado por un equipo de investigación del Instituto Valenciano de Investigaciones Económicas (IVIE) y de la fundación BBV, titulado El stock de capital en España y su distribución territorial ${ }^{29}$. El método utilizado por este grupo

26. Véase Valdaliso y López (2000). Esta conclusión también se deduce de los contratos de transferencia de tecnología que firmaron las empresas españolas en el período que nos ocupa, y que he podido estudiar hasta ahora (alrededor de unos 500).

El problema de las cifras que ofrece la contabilidad nacional para el caso del output en el período 1964-1973 es el cambio de metodología utilizada para su cálculo, así como el cambio de año base. Para una mayor explicación, véase Prados de la Escosura (1995), pp. 46-50.

${ }^{28}$ Véase Prados de la Escosura (1995).

${ }^{29}$ No se incluye en el presente trabajo el stock de capital público. 
de investigación para calcular el stock de capital. ha sido el del inventario permanente, usado en la mayoría de los estudios realizados en otros países. Este método permite estimar el stock de capital a partir de las series de inversión. El stock de capital bruto, siguiendo este procedimiento, se halla en función de los flujos pasados de inversión deducidos los retiros que hayan tenido lugar. Es decir,

$$
\begin{aligned}
A B_{t}^{i}= & A B_{t-1}^{i}+I B_{t}^{i}+R_{t}^{i} \\
R_{t}^{i}= & \sum_{j=0}^{M_{t}} r_{j}^{i} I B_{t ;}^{i} \\
A B_{t}^{i}= & \text { stock bruto de capital del activo } i \text { en } t . \\
I B_{t}^{i}= & \text { inversión bruta de } i \text { en } t . \\
R_{t}^{i}= & \text { retiros producidos en el periodo } t \text { del activo } i . \\
r_{j}^{i} & =\text { tasa de retiro del bien } i \text { después de } j-\mathrm{I} \text { períodos de haberse realizado } \\
& \text { la inversión. }
\end{aligned}
$$

A partir del stock de capital bruto, de las cifras de inversión bruta y de las tasas de consumo de capital fijo se obtiene el stock de capital neto:

$$
\begin{aligned}
& A N_{t}=A N_{t}-1 \times\left(1-\delta_{t}\right)+I B_{t}, \mathrm{~V} t \\
& A N_{t}=\text { stock de capital neto del activo } i \text { en el periodo } t \\
& \delta_{t}=\text { tasa de consumo de capital neto. }
\end{aligned}
$$

Los trabajos del IVIE ofrecen estimaciones del stock de capital neto y de la inversión bruta para los siguientes sectores ${ }^{30}$ : agricultura, pesca, productos energéticos, minerales metálicos y siderometalúrgicos, minerales y productos no metálicos, productos químicos, productos metálicos n. c. o. p., maquinaria agrícola e industrial, máquinas de oficina y otros, material y accesorios eléctricos, material de transporte, productos alimenticios, bebidas y tabaco; productos textiles, cuero, calzado y vestido; papel, artículos de papel e impresión; productos de caucho y otros; madera, corcho y otras manufacturas; construcción; residencial; hostelería y restauración; transporte; comunicaciones; instituciones de crédito y seguros; otros servicios destinados a la venta n. c. o. p.

Para estimar la participación del capital en la remuneración total de los factores, y bajo el supuesto de competencia perfecta y rendimientos constantes de escala, se supone que aquélla es igual a uno menos la participación estimada del trabajo. Dada la participación del capital, el stock

30) Fundación BBV (1998). 
de capital, las tasas de consumo de capital neto (calculadas a partir de la fórmula anterior del stock de capital neto y de los datos ofrecidos por el estudio del IVIE anteriormente citado) y el precio de los bienes de capital (calculados a partir de los datos ofrecidos por el trabajo del IVIE) se puede hallar la tasa de beneficio en el año $t, r(t)$, a partir de la siguiente fórmula:

$$
\begin{aligned}
v_{K}(t)=P_{K i}(t) K_{j}(t)= & \left\{P_{I i}(t-1) r(t)+\delta P_{I i}(t)-\left[P_{I i}(t)-P_{1}\right.\right. \\
& \left.\left.{ }_{i}(t-1)\right]\right\} A_{j}(t-1)
\end{aligned}
$$

El cálculo del precio de los servicios del capital, $P_{K_{i}}(t)$, se obtiene aplicando la fórmula:

$$
P_{K i}(t)=P_{I i}(t-1) r(t)+\delta P_{I i}(t)-\left[P_{l i}(t)-P_{l i}(T-1)\right]
$$

Esta ecuación muestra que, para un $P_{l i}$ dado, la fuente de variación en el precio del capital es la tasa de depreciación. Ceteris paribus, el capital con una vida más corta tiene un precio estimado más alto que el capital de una larga vida. Como ha afirmado Hulten, el tener en cuenta la hipótesis del cambio tecnológico implícito en el capital implica que un cambio hacia capitales nuevos es equivalente a una mejora en la calidad del capital ${ }^{31}$. Con los datos obtenidos de precios y cantidades obtenemos el índice Divisia - Translog del factor capital, definido anteriormente en la ecuación 5.

Para hallar el input trabajo se ha calculado el número de asalariados clasificados por sectores y categorías socioprofesionales. La fuente empleada ha sido la encuesta Salarios del INE. Los sectores estudiados son: agricultura, extracción de carbón, extracción de minerales metálicos, extracción de minerales no metálicos, alimentación, bebidas y tabaco; industrias textiles; fabricación de calzado y cuero; industrias de la madera y el corcho; fabricación de papel y artículos de papel; imprentas y editoriales; fabricación de productos de caucho; fabricación de derivados del petróleo, carbón y minerales no metálicos; industrias metálicas; construcción y obras públicas; electricidad, gas y agua; comercio; bancos y otros establecimientos financieros, y compañías de seguros. En cuanto a la población no asalariada en agricultura, industria y servicios, se han empleado los datos ofrecidos por la publicación periódica del Banco de Bilbao, La renta nacional y su distribución provincial ${ }^{32}$. En cuanto a las categorías laborales, la población

${ }^{31}$ Véase Hulten (1992), p. 965.

${ }_{2}$ Dado que el número de trabajadores que contempla la encuesta de Salarios es inferior al que registra la publicación del Banco de Bilbao, se ha mantenido idéntica proporción a la hora de contabilizar los trabajadores no asalariados en las distintas ramas de actividad. 
asalariada del sector industrial y del sector servicios se ha clasificado en: empleados y operarios. Dentro de los primeros se incluyen los técnicos titulados, otros técnicos, administrativos y subalternos. Los operarios engloban: los jefes de equipo y oficiales de $1 .^{\text {a }}$; los oficiales de $2 .^{a}$ y $3 .^{a}$; los peones especializados; peones; y los aprendices y pinches ${ }^{33}$. La fuente utilizada ha sido la publicación periódica del Instituto Nacional de Estadística denominada encuesta Salarios ${ }^{34}$.

En segundo lugar, el cálculo de las horas trabajadas por la población asalariada exige distribuir el número total de horas entre los asalariados distribuidos en las categorías y sectores definidos anteriormente. Este paso se ha realizado a partir de la encuesta Salarios. A los trabajadores no asalariados se les ha asignado el mismo número de horas trabajadas que a los asalariados del sector correspondiente. Con el número de trabajadores y el número total de horas trabajadas por trabajador calculamos el índice cuántico de trabajo. Para hallar las horas trabajadas por los trabajadores agrícolas se ha supuesto que se trabajan 250 días al año y ocho horas al día.

Para poder estimar el índice Translog del input trabajo se necesitan conocer, además, los precios de las distintas clases del mismo, esto es, los salarios de los empleados. La encuesta de salarios proporciona la retribución media mensual por hora trabajada en cada sector y categoría socioprofesional de los asalariados en la industria y los servicios. La estadística de salarios es la fuente de información principal para conocer la remuneración de los asalariados. La variable a estimar es el salario, que se define en la encuesta como «la remuneración total que percibe el trabajador, exceptuando lo percibido por dietas y plus familiar, así como la remuneración en especie» ${ }^{35}$. La encuesta tiene una periodicidad trimestral, pero en el presente trabajo se han tomado las medias anuales, con objeto de evitar las variaciones estacionales. Las series de salarios para cada sector se han calculado ponderando los salarios en las diferentes ramas productivas por las horas trabajadas en ellas. Para los trabajadores no asalariados se ha supuesto un salario igual al salario medio de un asalariado de su mismo

${ }^{33}$ Técnicos titulados: ingenieros, licenciados y peritos; otros técnicos: técnicos no titulados de oficina, laboratorio y taller; administrativos: jefes administrativos, oficiales administrativos, auxiliares y aspirantes administrativos; subalternos. Mano de obra cualificada: jefes de equipo y oficiales. Mano de obra no cualificada: peones, aprendices y pinches.

${ }^{34}$ La Encuesta de Salarios tiene algunas deficiencias, como es la no cobertura de gran parte de los trabajadores asalariados del sector servicios. Tan sólo contempla los asalariados del comercio, sector bancario y seguros.

${ }^{35}$ Salarios, año 1963, INE (1964). 
sector. En el caso de la agricultura, los salarios medios se han tomado de los datos ofrecidos por Jordi Maluquer de Motes que aparecen en el libro Estadisticas bistóricas de España (1989).

\section{CUADRO 1}

Tasas anuales de crecimiento del PIB, 1964-1973

\begin{tabular}{|c|c|c|c|c|c|c|c|c|c|c|}
\hline Años & Canadá & Francia & Alemania & Japón & Corea & Holanda & $\begin{array}{l}\text { Reino } \\
\text { Unido }\end{array}$ & $E E U U$ & Italia & España \\
\hline $1964 \ldots \ldots \ldots . .$. & 6,9 & 7,6 & 7,2 & 11,9 & 6,3 & 9,6 & 7,2 & 5,3 & 3,4 & 8,5 \\
\hline $1965 \ldots \ldots \ldots . . .$. & 7,1 & 5,4 & 6,3 & 9,5 & 7,1 & 6,2 & 2,5 & 6,0 & 3,4 & 6,1 \\
\hline $1966 \ldots \ldots \ldots . . .$. & 6,6 & 5,8 & 3,5 & 7,6 & 12,3 & 3,5 & 2,8 & 0,6 & 5,6 & 7,1 \\
\hline $1967 \ldots \ldots \ldots . . .$. & 2,9 & 5,0 & 0,0 & 11,4 & 8,4 & 4,9 & 3,1 & 2,7 & 7,3 & 6,2 \\
\hline $1968 \ldots \ldots \ldots . . .$. & 5,3 & 4,4 & 7,8 & 12,5 & 11,8 & 7,1 & 3,9 & 4,5 & 5,5 & 5,7 \\
\hline $1969 \ldots \ldots \ldots . . .$. & 4,3 & 8,1 & 7,0 & 11,3 & 17,7 & 6,7 & 1,0 & 3,1 & 6,1 & 8,8 \\
\hline $1970 \ldots \ldots \ldots \ldots$ & 3,8 & 6,1 & 7,1 & 12,3 & 7,4 & 6,7 & 3,7 & $-0,1$ & 5,3 & 5,6 \\
\hline $1971 \ldots \ldots \ldots . .$. & 5,0 & 4,7 & 3,1 & 9,3 & 8,7 & 5,2 & 3,4 & 3,5 & $-2,6$ & 6,0 \\
\hline $1972 \ldots \ldots \ldots \ldots$ & 5,0 & 5,4 & 4,4 & 8,4 & 8,9 & 6,2 & 5,1 & 6,3 & 2,8 & 8,2 \\
\hline $1973 \ldots \ldots \ldots .$. & 6,6 & 6,7 & 6,0 & 8,1 & 19,1 & 5,0 & 6,8 & 6,3 & 6,1 & 8,2 \\
\hline
\end{tabular}

FuENTE: Christensen, Cummings y Jorgenson (1980); calculado para España a partir de las series ofrecidas por Prados de la Escosura (1995).

\section{CUADRO 2}

Tasas anuales de crecimiento del input trabajo, 1964-1973

\begin{tabular}{crrrrrrrrrrr}
\hline Años & Canadá & Francia & Alemania Japon & Corea & Holanda & $\begin{array}{r}\text { Reino } \\
\text { Unido }\end{array}$ & EE UU & Italia & España \\
\hline $1964 \ldots . .$. & 3,1 & 1,3 & 1,2 & 3,0 & $-0,2$ & 1,3 & 1,8 & 2,1 & $-3,5$ & 1,8 \\
$1965 \ldots . .$. & 3,2 & $-0,6$ & $-0,8$ & 4,3 & 10,6 & 1,4 & $-0,2$ & 3,7 & $-6,9$ & 1,4 \\
$1966 \ldots . .$. & 2,7 & 1,2 & $-1,9$ & 2,7 & 3,0 & 1,1 & $-1,3$ & 3,8 & 2,7 & 4,6 \\
$1967 \ldots . .$. & 1,9 & $-0,2$ & $-5,8$ & 3,8 & 5,7 & $-1,8$ & $-1,5$ & 1,5 & 3,4 & 0,3 \\
$1968 \ldots .$. & 0,2 & $-0,7$ & 1,8 & 3,4 & 7,9 & 1,4 & 0,0 & 2,4 & 1,3 & $-2,2$ \\
$1969 \ldots . .$. & 1,9 & 1,7 & 1,6 & 1,4 & 6,3 & 1,6 & 0,5 & 3,2 & $-2,0$ & 0,6 \\
$1970 \ldots .$. & $-0,1$ & 0,8 & 0,6 & 1,8 & 0,2 & $-0,4$ & $-2,7$ & $-1,1$ & 1,9 & 0,7 \\
$1971 \ldots \ldots$. & 1,9 & $-0,2$ & $-2,2$ & 1,2 & 6,6 & $-0,1$ & $-5,3$ & 0,6 & $-2,5$ & $-0,2$ \\
$1972 \ldots \ldots$. & 2,7 & $-0,3$ & $-1,6$ & 1,4 & 6,4 & $-1,8$ & 1,0 & 3,8 & $-2,6$ & $-1,8$ \\
$1973 \ldots \ldots$. & 5,4 & 0,7 & $-0,3$ & 3,3 & 6,6 & $-0,4$ & 3,8 & 5,0 & $-0,4$ & $-1,0$ \\
\hline
\end{tabular}

Fuentl: Christensen, Cummings y Jorgenson (1980) para todos los países; para España, cálculos propios. 


\section{CUADRO 3}

Tasas anuales de crecimiento del input capital, 1964-1973

\begin{tabular}{cccccccccccc}
\hline Años & Canadá & Francia & Alemania Japón & Corea & Holanda & $\begin{array}{c}\text { Reino } \\
\text { Unido }\end{array}$ & EE UU & Italia & España \\
\hline $1964 \ldots \ldots$ & 4,5 & 6,6 & 6,7 & 8,9 & 3,9 & 6,3 & 4,6 & 3,9 & 7,8 & 6,0 \\
$1965 \ldots . .$. & 5,4 & 7,0 & 7,7 & 11,7 & 3,7 & 7,3 & 6,0 & 4,3 & 4,9 & 6,7 \\
$1966 \ldots \ldots$. & 6,5 & 6,1 & 8,4 & 8,9 & 3,1 & 7,4 & 5,1 & 5,3 & 3,6 & 5,7 \\
$1967 \ldots \ldots$. & 6,8 & 6,4 & 6,6 & 8,3 & 8,8 & 6,2 & 4,1 & 5,7 & 3,9 & 6,0 \\
$1968 \ldots \ldots$. & 5,4 & 6,0 & 4,1 & 11,7 & 11,3 & 5,7 & 4,3 & 4,4 & 5,2 & 7,1 \\
$1969 \ldots . .$. & 4,9 & 5,9 & 5,5 & 14,0 & 13,2 & 5,9 & 4,5 & 4,6 & 4,9 & 6,9 \\
$1970 \ldots \ldots$. & 5,1 & 6,7 & 7,1 & 13,8 & 12,7 & 6,0 & 3,4 & 4,6 & 5,3 & 5,0 \\
$1971 \ldots \ldots$. & 3,7 & 6,4 & 7,6 & 14,8 & 9,6 & 7,6 & 3,5 & 3,1 & 6,0 & 6,6 \\
$1972 \ldots \ldots$. & 4,4 & 6,2 & 7,3 & 12,3 & 9,0 & 6,7 & 4,7 & 3,5 & 4,9 & 8,6 \\
$1973 \ldots \ldots$. & 5,2 & 6,4 & 6,2 & 11,6 & 5,4 & 6,2 & 5,9 & 4,5 & 4,6 & 9,9 \\
\hline
\end{tabular}

FuENTE: Christensen, Cummings y Jorgenson (1980); para España, cálculos propios.

\section{CUADRO 4}

Tasas de crecimiento anuales de la productividad total de los factores, 1964-1973

\begin{tabular}{|c|c|c|c|c|c|c|c|c|c|c|}
\hline Años & Canada & Francia & Alemaria & Japón & Conea & Holanda & $\begin{array}{l}\text { Reino } \\
\text { Unido }\end{array}$ & $E E \cup U$ & Italia & España \\
\hline $1964 \ldots \ldots$ & 3,2 & 4,3 & 3,8 & 6,5 & 4,8 & 6,1 & 4,3 & 2,5 & 2,6 & 3,3 \\
\hline $1965 \ldots \ldots$ & 2,9 & 3,0 & 3,7 & 2,2 & $-0,6$ & 2,2 & 0,3 & 2,1 & 5,8 & 1,9 \\
\hline $1966 \ldots . .$. & 2,2 & 2,5 & 1,3 & 2,4 & 9,3 & $-0,3$ & 1,6 & 1,5 & 2,5 & 4,4 \\
\hline $1967 \ldots \ldots$ & $-1,2$ & 2,3 & 0,8 & 5,7 & 1,6 & 3,4 & 2,5 & $-0,6$ & 3,7 & 5,5 \\
\hline $1968 \ldots \ldots$. & 2,9 & 2,1 & 5,1 & 5,4 & 2,8 & 3,8 & 2,3 & 1,3 & 2,7 & 6,2 \\
\hline $1969 \ldots \ldots$ & 1,0 & 4,5 & 3,8 & 4,3 & 9,1 & 3,3 & $-1,0$ & $-0,7$ & 5,4 & 2,9 \\
\hline $1970 \ldots \ldots$ & 1,6 & 2,7 & 3,8 & 5,3 & 2,9 & 4,4 & 4,1 & $-1,3$ & 2,0 & 4,5 \\
\hline $1971 \ldots \ldots$ & 2,3 & 1,9 & 1,3 & 2,4 & 1,0 & 2,1 & 5,5 & 1,9 & $-3,1$ & 6,9 \\
\hline $1972 \ldots \ldots$ & 1,6 & 2,9 & 2,4 & 2,5 & 1,5 & 4,5 & 2,6 & 2,6 & 2,9 & 6,2 \\
\hline $1973 \ldots \ldots$ & 1,3 & 3,5 & 3,7 & 1,5 & 12,9 & 2,7 & 2,2 & 1,5 & 4,8 & 3,9 \\
\hline
\end{tabular}

FUENTL: Christensen, Cummings y Jorgenson (1980); para España, cálculos propios. 


\section{BIBLIOGRAFÍA}

Aвramovitz, M. (1993): «The Search for the Sources of Growth: Areas of Ignorance, Old and New», The Journal of Economic History, 53, 2, pp. 217-243.

BandRÉs, E. (1995): «Formación de capital», en J. L. GarCía Delgado (dir.), Lecciones de Economia Española, Madrid, Civitas, pp. 121-142.

BARro, R. J., y SAla I MARTín, X. (1992): «Convergence», Joumal of Political economy, 100 (2), pp. 223-251.

Barro, R. J., y Sala I Martin (1995): Economic Growth, New York, McGraw-Hill.

Baumol, W. J. (1986): «Productivity Growth, Convergence and Welfare: What the Long-Run Data Show», American Economic Review, 76, pp. 1072-1085.

Christensen, L. R.; Cummings, D., y Jorgenson, D. W. (1980): «Economic Growth, 1947-1973: An International Comparison», en J. W. KENDRICK y B. VACCARA (eds.), NBER Studies in Income and Wealth, 41, New York, Columbia University Press, pp. 595-698.

Comín, F. (1995): «La difícil convergencia de la economía española: un problema histórico», Papeles de Economía Española, 63, pp. 78-90.

DE LA FUENTE, A. (1998): Innovación tecnológica y crecimiento económico, Informe para la Fundación COTEC, Instituto de Análisis Económico, Barcelona.

DE LA FUENTE, A. (1995): «Inversión, "catch-up" tecnológico y convergencia real», Papeles de Economía Española, 63, pp. 18-34.

Denison, E. F. (1962): «The Unimportance of the Embodiment Question», American Economic Review, 54, pp. 90-94.

Diewert, W. E., y Nakamura, A. O. (1993): Essays in Index Number Theory, Amsterdam, North-Holland.

Dowrick, S., y NGUYEN, D. T. (1989): «OECD Comparative Economic Growth 1950-85: Catch-Up and Convergence», American Economic Review, 79, pp. 1010-1085.

Escribá Pérez, F. J., y Murgui García, M. J. (1998): «La productividad total de los factores entre sectores y regiones en la economía española (1980-1993)», Documento de trabajo D-98005, Dirección General de Análisis y Programación Presupuestaria, Ministerio de Economía y Hacienda.

FundaCión BBV (1999): La Renta Nacional de España y su distribución provincial: serie bomogénea, años 1955-1993, Madrid.

FUNDACIÓN BBV (1998): El stock de capital en España y su distribución territorial, M. Mas, F. Pérez y E. URiel (directores), Bilbao.

GreenwoOd, J.; Hercowitz, Z., y Krusell, P. (1998): «The Role of Investment-Specific Technological Change», American Economic Review, 87, pp. 342-362.

Grossman, G., y Helpman, E. (1991): Innovation and Growth in the Global Economy, MIT Press, Cambridge, MA.

Hulten, CH. (1992): «Growth Accounting when Technical Change is Embodied in Capital», American Economic Review, 82, 4, pp. 964-980.

Hulten, CH. (2000): «Total Factor Productivity: A Short Biography», Working Paper, 7471, Cambridge, MA., National Bureau of Economic Research.

InstTtuto Nacional de Estadtstica (varios años): Salarios, Madrid. 
Jorgenson, D. W.; y Griliches, Z. (1967): «The Explanation of Productivity Change», en D. W. Jorgenson (1996), Productivity, Cambridge, MIT Press, vol. 1, pp. 51-99.

Jorgenson, D. W., Gollop, F. M., y Fraumeni, B. (1987): Productivity and US Economic Growth, North-Holland, Amsterdam.

JoRGenson, D. W. (1996): «Productivity and Economic Growth», en D. W. JoRGENSON, Productivity, Cambridge, MIT Press, vol. 2, pp. 1-87.

LuCAS, R. E. (1990): «Why Doesn't Capital Flow from Rich to Poor Countries?», American Economic Review, 80, 1, pp. 92-96.

Maddison, A. (1987): «Growth and Slowdown in Advanced Capitalist Economies: Technique of Quantitative Assessment, Joumal of Economic Literature, 25, pp. 649-98.

MALUQUeR DE Motes, J. (1989): «Precios, salarios y beneficios. La distribución funcional de la renta», en A. CARRERAs (ed.), Estadísticas bistóricas de España, siglos $X I X$ y $X X$, Madrid, Fundación Banco Exterior.

MANKTW, N. G.; Romer, D., y WEIL, D. N. (1992): «A Contribution to the Empirics of Economic Growth», Quarterly Joumal of Economics, 107, 2, pp. 407-437.

Martínez Serrano, J. A., y Muñoz, C. (1995): «Sector Servicios», en J. L. Garcia Delgado (dir.): Lecciones de Economia Española, Madrid, Civitas, pp. 267-282.

Maudos, J.; PAStor, J. M., y SerRANo, L. (2000): «Crecimiento de la productividad y su descomposición en progreso técnico y cambio de eficiencia: una aplicación sectorial y regional en España (1964-93)», Investigaciones Económicas, 24, 1, pp. 177-205.

MYRO, R. (1983): «La evolución de la productividad global de la economía española en el período 1965-81», Información Comercial Española, febrero, pp. 115-127.

Myro, R., y GandoY, R. (1995): «Sector industrial», en J. L. Garcí Delgado (dir.), Lecciones de Economía Española, Madrid, Civitas, pp. 245-266.

Pérez, F.; Goerlich, F. J., y MAS, M. (1996): Capitalización y crecimiento en España $y$ sus regiones, 1955-1995, Madrid, Fundación BBV.

PhelpS, E. S (1962): «The New View of Investment: A Neoclassical Analysis», Quarterly Joumal of Economics, 76, pp. 548-567.

PRADOS DE LA EscosurA, L., y Zamagni, V. (eds.) (1995): El desarrollo económico en la Europa del Sur: España e Italia en perspectiva bistórica, Madrid, Alianza.

Prados de la Escosura, L. (1995): «Spain's Gross Domestic Product 1830-1993: quantitative conjectures», Working Paper, 95-05, Madrid, Universidad Carlos III.

- (1999): «El comportamiento de la economía española en términos comparados», Seminario de la UIMP, Formigal.

RAYMOND, J. L. (1995): «Crecimiento económico, factor residual y convergencia en los países de la Europa comunitaria», Papeles de Economía Española, 63, pp. 93-111.

ROMER, P. (1986): «Increasing Returns and Long-Run Growth», Journal of Political Economy, 94, 5, pp. 1002-1037.

SANCHIS LLOPIS, T. (1997): «Relaciones de intercambio sectoriales y desarrollo industrial. España, 1954-1972», Revista de Historia Industrial, 11, pp. 149-174.

Solow, R. M. (1957): «Technical Change and the Aggregate Production Function», Review of Economics and Statistics, 39, pp. 312-320, en B. VAN ARK (ed.), Eco- 
nonomic Growth in the Long Run: a History of Empirical Evidence, vol. II, Edgar Elgar, UK.

SuÁreZ BERnAldo DE Quirós, F. J. (1992): «Economías de escala, poder de mercado y externalidades», Investigaciones Económicas (Segunda época), 16, 3, pp. 411-441.

Valdaliso, J. M., y López, S. (2000): Historia económica de la empresa, Madrid, Crítica. 